\title{
Analysis and Simulation of Multi-target Echo Signals from a Phased Array Radar
}

\author{
Zhen Jia1,a, Rui Zhou² \\ ${ }^{1}$ Jiangsu Automation Research Institute, 222061 Lianyungang, China \\ ${ }^{2}$ Zhengzhou Technical College, 450121 Zhengzhou, China
}

\begin{abstract}
The construction of digital radar simulation systems has been a research hotspot of the radar field. This paper focuses on theoretical analysis and simulation of multi-target echo signals produced in a phased array radar system, and constructs an array antenna element and a signal generation environment. The antenna element is able to simulate planar arrays and optimizes these arrays by adding window functions. And the signal environment can model and simulate radar transmission signals, radar internal noise, clutter and jamming signals. These signals are combined linearly to generate the radar echo signal. The simulation results indicate that multi-target echo signals are completely overwhelmed by other signals, satisfying the needs of subsequent signal processing and data processing.
\end{abstract}

\section{Introduction}

In recent years, many countries have been generalizing the phased array radar technology vigorously. Compared to other radars, the phased array radar has unique advantages: rapid scan of antenna beams, varieties of beam shapes, composition of space powers, and powerful data processing system, as well as tracing multiple targets simultaneously. In the real application, performance evaluation of a radar system is known to be significantly important. However, the evaluation implemented with traditional methods become more difficult now, and the semi-physical or all-digital simulation method can therefore be employed to fulfill design and evaluation of the radar system [1].

The generation of radar echo signals is of great importance to radar simulation systems. There are some studies on simulating the radar echoes by combining different kinds of signals. Cui et al. [2] investigated the modeling and simulation of the target echo and $K$ distributed clutter echo for each radar member, and simulated radar echo signals by combining the target echo with Gauss noise or clutter echo. Zhao et al. [3] presented a radar video signal simulation method based on the pre-recorded radar video signal, and radar video signals were consist of target echo signals, clutters and noises. Generally, the number of targets searched by radar is more than one, i.e. the simulated radar echo signal always contains several targets. Qin et al. [4] investigated the radar echo waveform and analyzed the performances of the target and sea clutter signals, reporting that the compound signal waveform could simulate the radar system's echo signal well, by superposing the targets, $\mathrm{K}$ distribution clutter and white

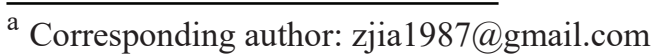

Gaussian noise signals. Wang et al. [5] studied and simulated the phased array radar system by using Simulink as the simulation platform, and their radar echo signal is the linear combination of target echo, Rayleigh distribution clutter and Gaussian white noise signals. However, few researches have been done on including jamming signals in the radar echoes.

In this paper, a signal generation environment is built as the front-end of a phased array radar simulation system. The target echo signal are investigated in detail, and noise, clutter and jamming signals are analyzed and simulated respectively. These four signals are ultimately combined to create radar echo signals, approximating the true radar returns.

\section{Target Echo Signal}

When a radar works, its signal transmitter will transmit a series of high-frequency and repeated periodic signal towards some specific space. If the signal encounters any target during the propagation course, the radar receiver will receive a return reflected by the target. Meanwhile, the receiver's internal noise will be mixed in the echo signal inevitably. The linear frequency modulated (LFM) signal is commonly used as the transmission signal. Because the matched filter used later is insensitive to the Doppler shift. Even if the echo signal has a larger frequency shift, the same filter can be applied to accomplish the pulse compression.

\subsection{Phase Array Antenna}

The reason why this radar is called the "phased array" radar is that its work basis is a phase-controlled array 
antenna. The array antenna has diversified forms, such as a linear array and a planar array. The planar array antenna can realize the antenna beam scan at the azimuth or pitch angle direction [7]. Both rectangular and circular array antennas can be simulated, and the rectangular array antenna is modeled here.

$E(\theta, \varphi)=\left|\frac{\sin \left[\left(N k d_{x} \sin \theta \cos \varphi\right) / 2\right]}{\sin \left[\left(k d_{x} \sin \theta \cos \varphi\right) / 2\right]}\right| \frac{\sin \left[\left(N k d_{y} \sin \theta \cos \varphi\right) / 2\right]}{\sin \left[\left(k d_{y} \sin \theta \cos \varphi\right) / 2\right]} \mid(1)$

Where $N$ is number of antenna array elements, $k$ is wave number, $d_{\mathrm{x}}$ is vertical spacing between array elements, $d_{\mathrm{y}}$ is horizontal spacing between array elements, and $\theta$ is azimuth angle of the antenna beam pointing, as well as $\varphi$ is pitch angle of the antenna beam pointing. When simulating, all supposed parameters of the radar antenna are listed in Table 1 , where $\lambda$ is the radar working wavelength. The simulation results are shown in Figure 1.

Table 1. Total parameters of the phased array antenna

\begin{tabular}{|c|c|c|c|}
\hline Parameter & Value & Parameter & Value \\
\hline $\mathrm{N}$ & $30 \times 30$ & $k$ & $2 \pi / \lambda$ \\
\hline$d_{\mathrm{x}}$ & $0.5 \lambda$ & $\theta$ & $30^{\circ}$ \\
\hline$d_{\mathrm{y}}$ & $0.5 \lambda$ & $\varphi$ & $45^{\circ}$ \\
\hline
\end{tabular}

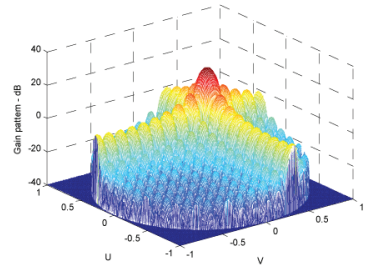

(a)

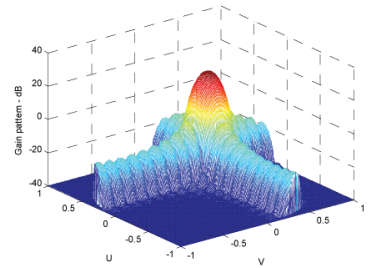

(b)
Figure 1. Antenna direction pictures of the rectangular array. (a) without adding a window; (b) after adding a Kaiser window.

Seen from Figure 1(a), the first side lobe is about $13 \mathrm{~dB}$ lower than the main lobe. This is far from adequate for most radar applications, but window functions can be added to reduce the side lobe level. The common window functions are rectangular, Bartlett, Blackman, Hamming, Hanning and Kaiser windows [7]. Here the Kaiser window is added, and its effect is demonstrated in Figure 1(b). It is clear that, the side lobe amplitude is reduced and the main lobe is widened.

\subsection{Radar Noise}

In the simulation process, the noise of the phased array radar itself is treated as a Gaussian white noise. And this noise should also be added to the received echo signal, which can be produced through a built-in random function of MATLAB.

In the paper, three point targets are supposed to be searched by the phased array radar. Table 2 lists their own initial movement parameters. The target range can be transformed to a range gate, and the target velocity conversed to a Doppler shift. Figure 2(a) shows the simulated multi-target echo signal, and Figure 2(b) is the echo signal mixed by the radar's internal noise.
Table 2. The initial parameters of three targets

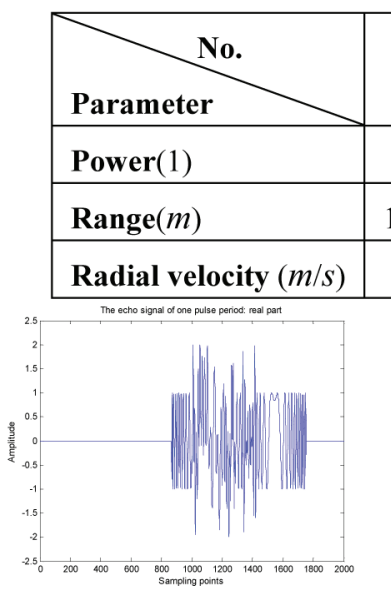

(a)

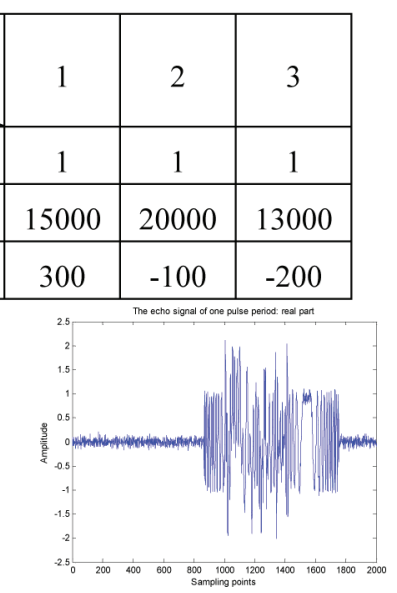

(b)
Figure 2. Time domain waveform of target echo signals. (a) without the white noise; (b) with the white noise.

\section{Clutter}

The clutter in a resolution unit contained a number of scatterers. The phase and amplitude of these scatterers are stochastic, and probability distribution functions can therefore be leveraged to describe the clutter statistically [7-9]. The types of these functions are determined by characters of the clutter itself, grazing angle and radar operating frequency.

\subsection{Rayleigh Distribution Clutter}

If a sea or ground clutter contains a lot of scatterers, and the probability of one scatterer receiving the return is not relevant to that of another scatterer, the clutter can be modeled with the Rayleigh distribution:

$$
f(x)=\frac{x}{\sigma^{2}} \exp \left(-\frac{x^{2}}{2 \sigma^{2}}\right), \quad x \geq 0
$$

where $\sigma$ is standard error of the clutter.

\subsection{Lognormal Distribution Clutter}

The lognormal distribution is good at describing the ground clutter with a low grazing angle, as well as depicting the high-resolution radar sea clutter [4]. It can be expressed as:

$$
f(x)=\frac{1}{\sqrt{2 \pi} \sigma_{c} x} \exp \left[-\frac{\ln ^{2}\left(x / u_{m}\right)}{2 \sigma_{c}^{2}}\right], \quad x>0, \sigma_{c}>0, u_{m}>0
$$

where $u_{\mathrm{m}}$ is scale parameter, representing the distribution mean; $\delta_{\mathrm{c}}$ is form parameter, meaning the distribution gradient.

\subsection{Weibull Distribution Clutter}

The Weibull distribution can model the clutter whose frequency is between $1 \sim 10 \mathrm{GHz}$ and grazing angle below $5^{\circ}[5]$. This model lies between the Rayleigh distribution and the lognormal distribution, and its formula is as following: 


$$
f(x)=\frac{p}{q}\left(\frac{x}{q}\right)^{p-1} \exp \left[\left(-\frac{x}{q}\right)^{p}\right], \quad x \geq 0
$$

where $p$ is form parameter, with a value $0<p \leqslant 2 ; q$ is scale parameter. Noting that the Weibull distribution will turn into the Rayleigh distribution when $p=2$.

\subsection{K Distribution Clutter}

A large number of analyses on actual measurement data indicate that, the sea clutter not only is a simple stationary stochastic process, but also has some fast and slow modulated variables [6]. By curve fitting, it was proved that the correlated $K$-distribution is able to describe the high-resolution radar sea clutter, whose probability density function is:

$$
f(x)=\int_{0}^{\infty} p(y) p\left(\frac{x}{y}\right) d y=\frac{2 c}{\Gamma(v)}\left(\frac{c x}{2}\right)^{v} K_{v-1}(c x)
$$

where $K_{v-1}(\cdot)$ is $(v-1)$ order second revised Bessel function; $c$ is proportion coefficient, with a value $\sqrt{\frac{v \pi}{E\left(y^{2}\right)}}$, in which $E\left(y^{2}\right)$ is average clutter power.

There are three steps to generate the above-mentioned four clutters: (1) Generating a Gaussian white noise sequence; (2) Processing the white noise via a linear filter, and gaining a correlated Gaussian sequence; (3) Generating a correlated random sequence with arbitrary distribution, by adopting the zero memory nonlinearity (ZMNL) or spherically invariant random processing (SIRP) method [7]. The simulation results of these clutters are shown in Figure 3.

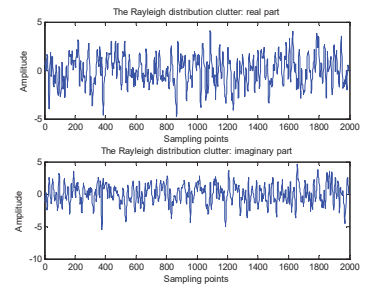

(a)

(c)

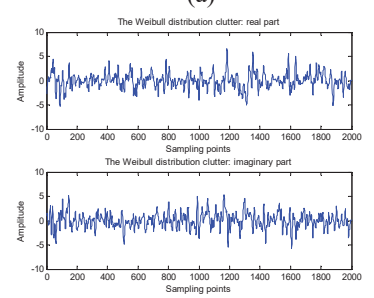

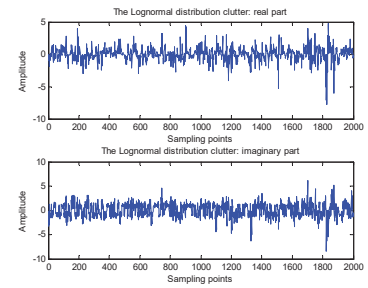

(b)

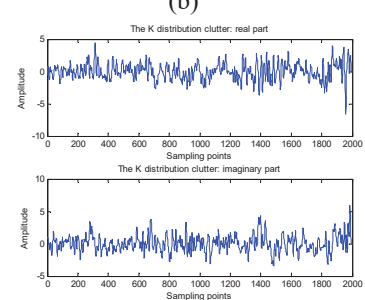

(d)
Figure 3. Time domain waveform of clutter signals. (a) Rayleigh distribution; (b) Lognormal distribution; (c) Weibull distribution; (d) $K$ distribution.

\section{Jamming}

As one of the important radar countermeasure's components, radar jamming is the technical name of destroying and hindering normal operation of the enemy' electronic and radar equipments. It makes the radar unable to detect and trace the true targets correctly, in order to disturb the radar's working. In terms of jamming energy source, radar jamming falls into unintentional and intentional jammings. And intentional jamming is divided into active and inactive jammings based on transmitting electromagnetic signals or not. Moreover, active jamming splits into masking and deception jammings [7,10-12].

\subsection{Masking Jamming}

Masking jamming can overwhelm or cover the target signals by using noise or noise-like jamming signals, to hinder the radar to detect target information. Its basic principle is use the radar to detect the target echo between external and internal noises, and the detecting process can be carried out with certain guidelines: If the SNR exceeds the detection threshold, targets were considered to exist; if not, there are no targets. According to producing way of the masking noise signals, active masking jamming can be classified into four kinds below.

\subsubsection{RF Noise Jamming}

The jamming that magnifies and transmits the narrowband Gaussian noise directly is RF noise jamming, also called direct noise amplifier (DINA). The expression of narrowband Gaussian noise is:

$$
J(t)=U_{n}(t) \cos \left(\omega_{j} t+\varphi\right)
$$

where envelope function $U_{\mathrm{n}}(t)$ obeys the Rayleigh distribution; phase function $\varphi(\mathrm{t})$ obeys the $[0,2 \pi]$ uniform distribution, independent of $U_{\mathrm{n}}(t)$; carrier frequency is a constant and much larger than the bandwidth of $J(t)$, and $J(t)$ is generally formed through filtration and amplification of a low-power noise.

\subsubsection{Noise AM Jamming}

The carrier's oscillation amplitude varies with the change of modulated noise, and this modulation process is called noise AM. The signal's expression is:

$$
J(t)=\left[U_{j}+U_{n}(t)\right] \cos \left(\omega_{j} t+\varphi\right)
$$

where $U_{\mathrm{j}}$ is a constant.

\subsubsection{Noise FM Jamming}

If the carrier's instant frequency changes with modulation voltage, while the amplitude holds the line, this circumstance is referred to as noise FM. The signal expression is:

$$
J(t)=U_{j} \cos \left[\omega_{j} t+2 \pi k_{F M} \int_{0}^{t} U_{n}(t) d t+\varphi\right]
$$

where $k_{\mathrm{FM}}$ is FM slope.

\subsubsection{Noise PM Jamming}

The expression of a generalized stationary random noise PM process is:

$$
J(t)=U_{j} \cos \left[\omega_{j} t+k_{P M} U(t)+\varphi\right]
$$

where $k_{\mathrm{PM}}$ is a constant, meaning PM slope. Figure 4 represents time domain waveform and frequency 
spectrogram of the above-mentioned masking jamming signals.

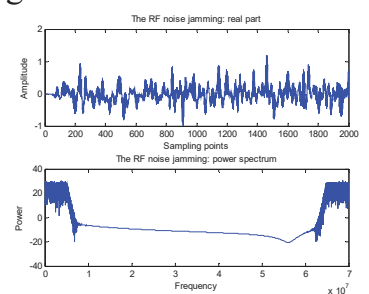

(a)

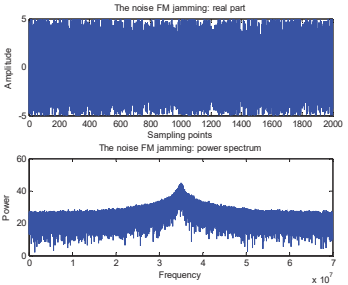

(c)

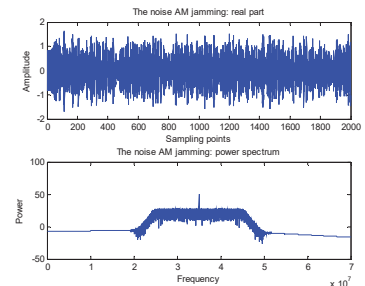

(b)

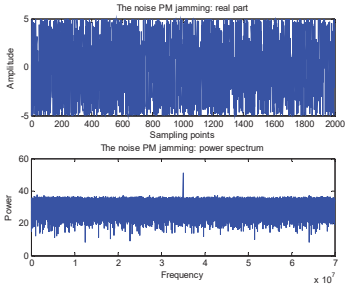

(d)
Figure 4. Time domain waveform and spectrogram of masking jamming signals. (a) RF noise jamming; (b) noise AM jamming; (c) noise FM jamming; (d) noise PM jamming.

\subsection{Deception Jamming}

The basic principle of deception jamming is produce one jamming signal to act on the radar, which has similar features as the transmission signal, in order to interrupt the enemy to detect the true targets' signal parameters. This way will disturb the radar system on an information level. Here, range and velocity deception jammings are studied by theoretical analysis and simulation.

\subsubsection{Range False Target Jamming}

The main theory of range false target jamming is delay and amplify the received radar transmission signal, aimed to disturb the target detection. Suppose the distance between a true target and the radar is $\mathrm{R}$, and time delay of the received echo is $\tau=2 R / c$; the distance between a false target and the radar is Rf, and time delay is $\tau=2 R_{\mathrm{f}} / c$. If

$$
\left|R_{f}-R\right|>\varepsilon R
$$

Then this false target is a range false one. If using the jamming theory of range false targets, and adopting the pulse delay stacking technique, many false targets will be produced to interrupt the radar to detect the true targets normally.

\subsubsection{Range Pull-off Jamming}

The false target range function $R_{\mathrm{f}}(t)$ of range pull-off jamming can be expressed as:

$$
R_{f}(t)=\left\{\begin{array}{ccc}
R & 0 \leq t<t_{1} & \text { Stop } \\
R+v\left(t-t_{1}\right) \text { or } R+a\left(t-t_{1}\right)^{2} & t_{1} \leq t<t_{2} & \text { Pull off } \\
\text { Jamming Shutoff } & t_{2} \leq t<T_{j} & \text { Closed }
\end{array}\right.
$$

where $R$ is target distance; $v$ is velocity when pulled off at a constant speed, and $a$ is acceleration when pulled off at a constant accelerated speed.

Under the self-defense jamming condition, in which $R$ is also target distance, the equation above can be converted into time delay of the received radar project signal retransmitted by the jammer. The retransmitted time delay of range pull-off jamming $\Delta t_{\mathrm{f}}$ is

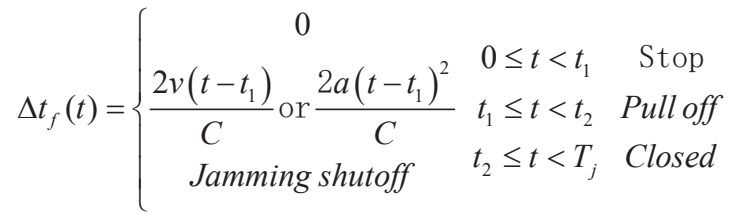

Here the uniform pull-off way is utilized to disturb the true targets, and Figure 6(b) shows the time domain waveform of range pull-off jamming.

\subsubsection{Velocity False Target Jamming}

The typical velocity false target jamming is the frequency-shift stacking jamming, whose principle is that the target's Doppler frequency measured by radar is influenced by the jamming radar's receiver. Learning from Fourier transform, frequency shift is time domain of the original signal multiply $e^{-\mathrm{j} 2 \pi \mathrm{ft}}$. The following jamming signal can be generated from the LFM signal.

$$
J(t)=A u(t) \sin \left\{2 \pi\left[\left(f_{0}+\Delta f\right) t+\frac{1}{2} k t^{2}\right]\right\}
$$

If the shift frequency is positive $(\triangle f>0)$, the false target is ahead of the true target; otherwise, the false target falls behind.

\subsubsection{Velocity Pull-off Jamming}

The basic theory of velocity pull-off jamming is retransmit the jamming signal with the same Doppler frequency $f_{\mathrm{d}}$ as the target return, and the energy of the jamming signal is higher than that of the target return, to enable the radar's speed tracking circuit to capture the Doppler frequency fd. Because the jamming energy is higher, the speed tracking circuit will follow by the jamming's Doppler frequency, resulting in velocity information errors. In the velocity pull-off jamming, Doppler frequency $f_{\mathrm{dj}}$ of the jamming signal is expressed as following:

$$
f_{d j}(t)=\left\{\begin{array}{ccc}
f_{d} & 0 \leq t<t_{1} & \text { Stop } \\
f_{d}+v_{f}\left(t-t_{1}\right) & t_{1} \leq t<t_{2} & \text { Pull off } \\
\text { Jamming shutoff } & t_{2} \leq t<T_{j} & \text { Closed }
\end{array}\right.
$$

where $v_{\mathrm{f}}$ is change rate of the interfering frequency, namely the separation velocity, whose plus or minus depends on acceleration direction (i.e. pull-off direction) of the velocity pull-off jamming. The time domain waveforms of the above-mentioned deception jamming signals are shown in Figure 5.

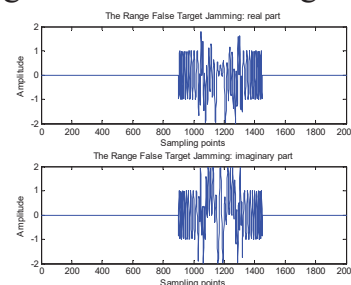

(a)

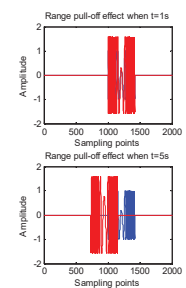

(b)

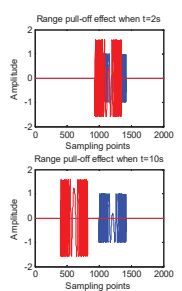




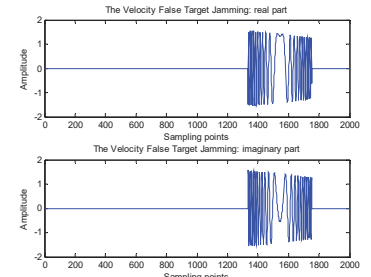

(c)

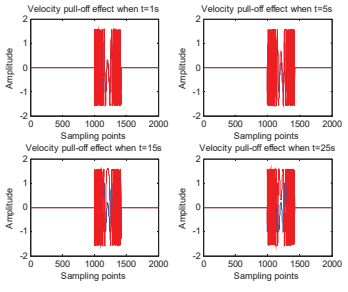

(d)
Figure 6. Time domain waveform of deception jamming signals. (a) range false target jamming; (b) range pull-off jamming; (c) velocity false target jamming; (d) velocity pull-off jamming.

\section{Radar Echo Signal}

The radar echo signal is achieved by superposing the target echo signal, noise, clutter and jamming linearly, as shown in Figure 6. There are several types of clutter and jamming signals simulated in the paper, therefore two optional combinations are used to construct the multitarget echo signal. The first one is the combination of radar transmission signal, Gaussian white noise, Rayleigh distribution clutter and noise AM jamming. The second one is the combination of radar transmission signal, Gaussian white noise, $K$ distribution clutter and range false target jamming. These two combinations are respectively shown in Figure 7.

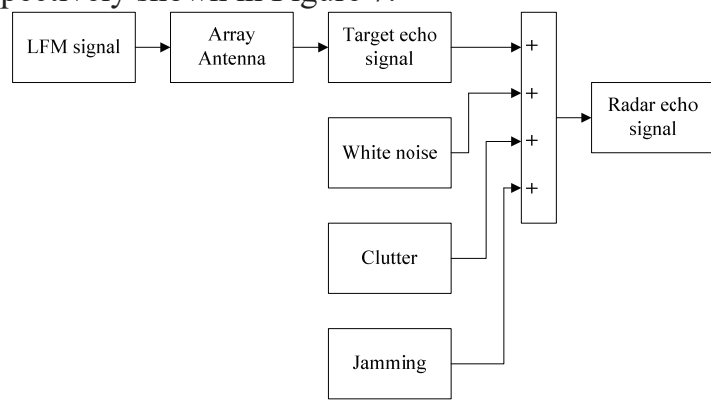

Figure 6. Linear combination for the radar echo signal.

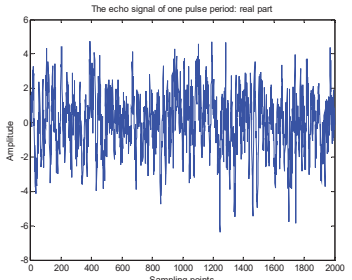

(a)

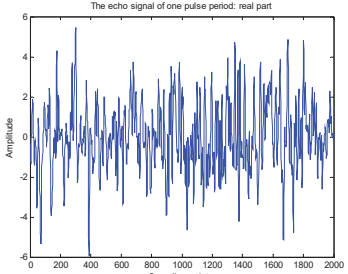

(b)
Figure 7. Time domain waveform of the radar echo signal. (a) first combination; (b) second combination.

Comparing with Figure 2, it can be seen that target signals are totally overwhelmed by noise, clutters and jammings, and they cannot be distinguished intuitively. Therefore, the signals are considered to approximate the true radar returns.

\section{Conclusion}

In the paper, with the purpose of attaining multi-target radar echo signals, the transmission signal, noise, clutter and jamming are analyzed theoretically and simulated in MATLAB. After superposing these four signals, the resulting return signal is significantly different from the transmission signal. In the future, signal processing and data processing modules of the phased array simulation system will be constructed, in order to complete range and velocity information measurements, and display multi-target movements on the radar search interface.

\section{References}

1. S.H. Talisa, K.W. O’Haver, T.M. Comberiate, et al. Benefits of Digital Phased Array Radars. Proceedings of IEEE, 2016, 104(3): 530-543.

2. Xiaohai Cui, Tao Zeng, Teng Long. Radar Target Echo Signal and Clutter Simulator Based on HLA. 2004

3. Jianhe Zhao, Guoqing Qi, Xianying Song. Simulation of Marine Radar Video Signal. IEEE International Conference on Instrumentation and Measurement, Computer, Communication and Control, 2014: 805-808.

4. Hua Qin, Hao Cha, Mo Zhou, et al. Simulation and Modeling of Radar Echo Signal. IEEE International Conference on Fuzzy Systems and Knowledge Discovery, 2012: 1954-1957.

5. Taotao Wang, Xiaodong Wan, Jie He. Simualtion of Phased Array Radar Systems. Computers and Modernization, 2014, 2(47): 209-218.

6. M.U. Afzal, A.A. Qureshi, M.A. Tarar, et al. Analysis, Design, and Simulation of Phased Array Radar Front-end. Emerging Technologies (ICET), 2011, (7): 1-6.

7. B.R. Mahafa. Radar Systems Analysis and Design Using MALAB, Third Edition. 2013: 559-586.

8. Fuchun Zhang, Yanzhong Hao, Yongxue Liu, et al. Studies of Modeling and Simulation of Phased-Array Radar System. IEEE Internationl Conference on Cyber Technology in Automation, Control, and Intelligent Systems, 2012: 415-418.

9. C.D. Curtis, M. Yeary, J.L. Lake. Adaptive Nullforming to Mitigate Ground Clutter on the National Weather Radar Testbed Phased Array Radar. IEEE Transactions on Geoscience and Remote Sensing, 2016, 54(3): 1282-1291.

10. Kelei Wei, Ning Tai, Lei Huang, et al. Shiftfrequency jamming against pulse compression radar. 2016 Progress in Electromagnetic Research Symposium (PIERS), Shanghai, 2016: 2251-2254.

11. F. Novillo, J.R. Arguello, E. Guerrero, et al. Performance Evaluation of Radar Systems in Noise Jamming Enviroments. 2016 IEEE Ecuador Technical Chapters Meeting (ETCM), Guayaquil, 2016: 1-6.

12. S. Lu, G. Cui, W. Yi, et al. Radar Waveform Design against Signal-dependent Jamming. 2017 IEEE Radar Conference (RadarConf), Seattle, WA, USA, 2017: 1075-1080. 